\title{
Insomnia in neurological diseases
}

Geert Mayer ${ }^{1,2^{*}}$, Svenja Happe ${ }^{3}$, Stefan Evers ${ }^{4}$, Wiebke Hermann ${ }^{5}$, Sabine Jansen ${ }^{6}$, Ulf Kallweit ${ }^{7}$, Maria-Lucia Muntean ${ }^{8,9}$, Dieter Pöhlau ${ }^{9}$, Dieter Riemann ${ }^{10}$, Michael Saletu ${ }^{11}$, Melanie Schichl', Wolfgang J. Schmitt ${ }^{12}$, Friederike Sixel-Döring ${ }^{13}$ and Peter Young ${ }^{14}$

\begin{abstract}
Insomnia is defined as difficulties of initiating and maintaining sleep, early awakening and poor subjective sleep quality despite adequate opportunity and circumstances for sleep with impairment of daytime performance. These components of insomnia - namely persistent sleep difficulties despite of adequate sleep opportunity resulting in daytime dysfunction - appear secondary or co-morbid to neurological diseases. Comorbid insomnia originates from neurodegenerative, inflammatory, traumatic or ischemic changes in sleep regulating brainstem and hypothalamic nuclei with consecutive changes of neurotransmitters. Symptoms of neurological disorders (i.e motor deficits), co-morbidities (i.e. pain, depression, anxiety) and some disease-specific pharmaceuticals may cause insomnia and/or other sleep problems.

This guideline focuses on insomnias in headaches, neurodegenerative movement disorders, multiple sclerosis, traumatic brain injury, epilepsies, stroke, neuromuscular disease and dementia.

The most important new recommendations are: Cognitive behavioral therapy (CBTi) is recommended to treat acute and chronic insomnia in headache patients. Insomnia is one of the most frequent sleep complaints in neurodegenerative movement disorders. Patients may benefit from CBTi, antidepressants (trazodone, doxepin), melatonin and gaba-agonists. Insomnia is a frequent precursor of MS symptoms by up to 10 years. CBTi is recommended in patients with MS, traumatic brain injury and. Melatonin may improve insomnia symptoms in children with epilepsies. Patients with insomnia after stroke can be treated with benzodiazepine receptor agonists and sedating antidepressants. For patients with dementia suffering from insomnia trazodone, light therapy and physical exercise are recommended.
\end{abstract}

Keywords: Insomnia, Neurological diseases, Comorbid diseases, Classifications, Diagnostic instruments, Therapeutic recommendations, Cognitive behavioral therapy

\section{Introduction}

Insomnias with difficulties of initiating and maintaining sleep, excessive daytime sleepiness, motor disorders during sleep and parasomnias, early awakening and impaired sleep quality frequently accompany neurological diseases as secondary or comorbid conditions. The underlying causes of

\footnotetext{
* Correspondence: geert.mayer@t-online.de

'Neurologische Abteilung der Hephata-Klinik, Schimmelpfengstrasse 6, 34613

Schwalmstadt-Treysa, Germany

${ }^{2}$ Neurologische Abteilung der Philipps-Universität Marburg, Mamburg,

Germany

Full list of author information is available at the end of the article
}

many insomnias have not been fully elucidated, yet. Secondary insomnia may originate from neurodegenerative, inflammatory, traumatic or ischemic damage in sleep regulating brainstem and hypothalamic nuclei with consecutive changes of neurotransmitters. Symptoms of neurological disorders (i.e. motor deficits), co-morbidities (i.e. pain, depression, anxiety) and some specific medication result in insomnia and/or other sleep problems. Insomnias have a strong impact on quality of life, cognition and physical well-being and therefore need special consideration for diagnosis and therapy.

(c) The Author(s). 2021 Open Access This article is licensed under a Creative Commons Attribution 4.0 International License, which permits use, sharing, adaptation, distribution and reproduction in any medium or format, as long as you give appropriate credit to the original author(s) and the source, provide a link to the Creative Commons licence, and indicate if changes were made. The images or other third party material in this article are included in the article's Creative Commons licence, unless indicated otherwise in a credit line to the material. If material is not included in the article's Creative Commons licence and your intended use is not permitted by statutory regulation or exceeds the permitted use, you will need to obtain permission directly from the copyright holder. To view a copy of this licence, visit http://creativecommons.org/licenses/by/4.0/. 
This guideline focuses on insomnias in headaches, neurodegenerative movement disorders, multiple sclerosis, traumatic brain injury, epilepsies, stroke, neuromuscular disease and dementia.

\section{Classification and definitions}

The ICD 10/11, ICSD 3 and DSM V differentiate as "transient" or "chronic" insomnia. The ICD 11 contains a special chapter on sleep disorders, in which insomnia disorder is differentiated into short-term, chronic and unspecified insomnia disorders.

Chronic insomnia disorder is defined by the following diagnostic criteria (ICD 10):

A. Patient reports or caretaker observes

1. Difficulty initiating sleep

2. Difficulty maintaining sleep

3. Waking up earlier than desired

4. Resistance to going to bed on appropriate schedule

5. Difficulty sleeping without parent or caregiver intervention

B. The sleep disorder causes significant clinical suffering or impairment in social-, educational- and professional life or other important domains

C. The sleep disturbance and associated daytime symptoms occur at least three times per week

D. The sleep disturbance and associated daytime symptoms have been present for at least 3 months

E. The sleep disorder occurs despite sufficient opportunity to sleep

F. The insomnia is not attributed more clearly by another sleep disorder

G. The insomnia is not caused by the physiological effects of a substance

$\mathrm{H}$. The co-existing psychological and physical diseases do not explain the insomnia. If criteria for insomnia are met in context with a psychological or physical disease, both disorders will be diagnosed

In contrast to situational and transient insomnias defined as insomnia symptoms attributed to a certain event/situation or occurring transiently (meaning less than 3 months), occurring frequently without the need of specific interventions, chronic insomnias require specific treatment due to its impact on sleep quality, daytime performance and quality of life. According to ICD-10 [1] a diseaseworthy insomnia is given, when symptoms persist for 4 weeks. According to DSM-5 [2] disease duration of 3 months is required, which is then called chronic insomnia. Insomnia in neurological diseases is also classified according to ICD-10 [1].

\section{Diagnostics}

According to ICD 10/11 [1] the diagnosis of insomnia is based on clinical evaluations. Questionnaires may be added to complete evaluation of clinical symptoms within the diagnostic workup. For some of the neurological diseases, specific questionnaires addressing insomnia/sleep disturbances were designed, which are cited in the respective chapters. The recommended diagnostic work-up steps are as follows:

\author{
Medical history/diagnostic work-up \\ Psychiatric/psychological history \\ Sleep history \\ Actigraphy \\ Polysomnography
}

\section{Recommendations}

- The diagnostic work-up should comprise a complete medical history including physical and mental diseases, a physical examination, sleep questionnaires and sleep diary.

- The use of substances known to compromise sleep quality should be assessed thoroughly.

- Actigraphy may be used to evaluate bed and sleep times across $24 \mathrm{~h}$ and for longer time periods of days and weeks to assess circadian rhythm

- Polysomnography should be performed in cases suspicious for underlying organic sleep disorders (e.g., periodic leg movements, sleep related breathing disorders)

- Polysomnography is recommended

- in therapy-refractory insomnia

- after the above mentioned diagnostic steps to rule out secondary causes for insomnia have been performed

- if insomnia potentially endangers patients themselves or others

- in case of discrepancies between the subjective perception of insomnia and clinical and objective evaluations

\section{Therapeutic principles}

4.1. In general, patients sleep hygiene should be evaluated. Informations on improving sleep hygiene (psychoeducation) should be conveyed. Strategies to improve day-structuring should be considered. 
4.2. Flow chart

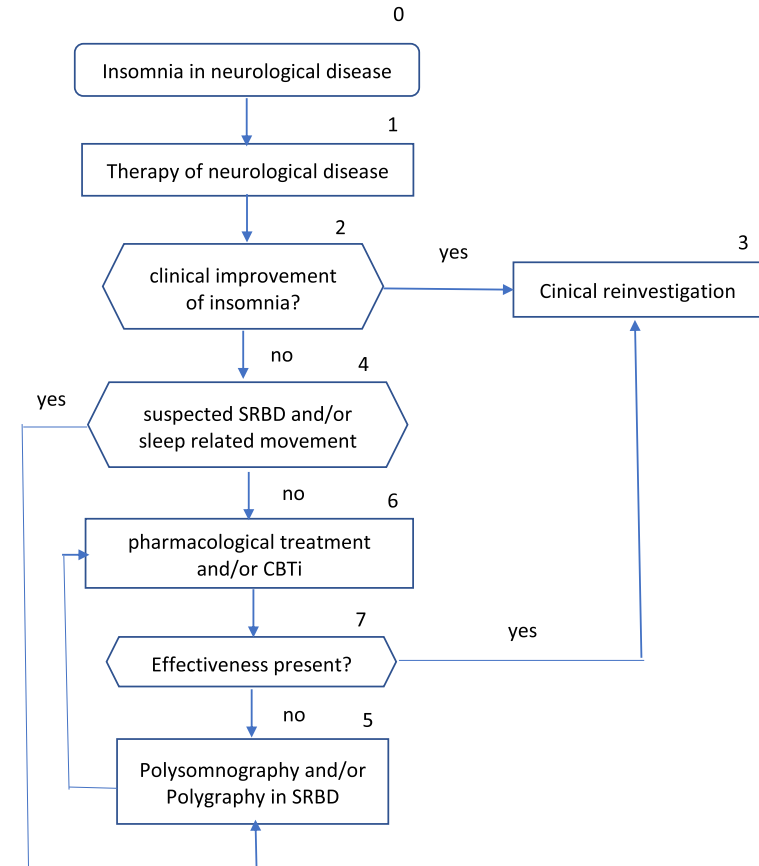

Insomnias comorbid to neurological disease should be treated according to international insomnia guidelines.

Collaboration with a certified sleep specialist is recommended for diagnostic work-up and treatment of insomnias. If polysomnography is indicated, the sleep laboratory should be accredited by the national sleep society and should be experienced in diagnosis and treatment of insomnias.

\section{Insomnia in neurological diseases}

The following chapter presents insomnias in the most common neurological diseases. Insomnia in restless legs syndrome and narcolepsy are covered in the respective European and American guidelines.

\section{Insomnia in headaches}

The International Headache Society (IHS) differentiates more than 200 forms of headaches in the International Classification of Headache Disorders, which are mainly unrelated to sleep. However, sleep related headaches usually emerge during sleep and may be accompanied by insomnia (according to ICSD3 [35]). These include different types of headaches such as migraine, cluster headache, chronic paroxysmal hemicrania $(\mathrm{CPH})$ and hypnic headache syndrome. All of these forms show a close relationship between onset of pain and sleep and are listed separately in appendix A of the ICSD3 (ICSD-32014).

In a large Korean population-based evaluation, migraine was reported in $46 \%$ and other headache forms in $33 \%$ of patients with impaired sleep duration, whereas only $20 \%$ of controls suffered from headaches [6].
Similarly, migraine frequency increased in patients with short compared to longer sleep duration [7, 8].

In polysomnography study about $50 \%$ of patients with chronic tension headache and chronic migraine insomnia reported insomnia, but objective sleep parameters did not differ significantly from those without insomnia [9]. Interestingly, patients with the diagnosis of tension headache showed an increased amount of slow wave sleep with only few arousals in a Norwegian polysomnography based study, [10]. In an American population-based evaluation, insomnia was associated with overuse of medication in $44 \%$ of headache patients [11].

In a pilot trial 31 patients with either chronic insomnia or co-morbid (secondary) insomnia received either chronic behavioral therapy (CBTi) twice per week for 30 min (including advice on combined sleep hygiene and sleep restriction regimen) or unspecific advice on life style adjustments [12]. After 2 weeks the Intention-toTreat analysis showed a reduction of headache frequency of $26,9 \%$ in the CBTi group and $36,2 \%$ in the control group. The follow-up examination after 6 weeks of treatment showed a frequency reduction of headache episodes of $48 \%$ in the CBTI group and $25 \%$ in the control group. Significant improvement of objective sleep efficiency as assessed by actimetry as well as subjective sleep quality was detectable in the CBTi condition compared to the control group. In a controlled,double-blind pilot trial (RCT) 79 patients with migraine were randomized 1:1 to receive either $3 \mathrm{mg}$ eszopiclone or placebo at bedtime demonstrating significantly higher sleep efficiency and total sleep time as well as lower wake time frequency of nocturnal awakenings in the eszopiclone group [13].

\section{Recommendations}

- Besides symptom-oriented drug therapy, combined drug and CBT-I is recommended.

- Eszopiclone may be administered for at least 6 weeks in migraine patients with insomnia, since it does not have any influence on frequency, duration and intensity of migraine attacks.

\section{Insomnia in neurodegenerative movement disorders}

The recommendations are restricted to Parkinson's disease (PD), atypical Parkinson syndromes, and spinocerebellar ataxia (SCA).

Sleep disorders in neurodegenerative diseases affect up to $90 \%$ of all patients. Sleep disorders in neurodegenerative diseases can precede motor symptoms by years and may exhibit unfavorable impact on quality of life and cognition [14]. Insomnia is the most frequently 
associated sleep disorder [15]. 35-60\% fulfill the criteria of chronic insomnia [15].

To screen for sleep problems and score their severity in PD the task force of the Movement Disorder Society (MDS) recommends the use of the following scales and questionnaires: Parkinson's disease sleep scale (PDSS), Pittsburgh sleep quality index (PSQI) and SCOPA-Sleep [16].

Most studies on possible treatment options for insomnia in movement disorders have been performed in $\mathrm{PD}$ patients. Currently, there are no RCT treatment trials available addressing insomnia in supranuclear gaze palsy (PSP), corticobasal degeneration (CBD) or multisystem atrophy (MSA). The Task Force of the Movement Disorder Society for evidence-based medicine concludes that due to lack of studies no specific pharmacotherapy can be recommended for long-term treatment of sleep disorders in PD at present [17]. However, case series and small randomized studies report partially positive effects of some drugs. The European Federation of Neurological Societies/Movement Disorder Society for the therapy of insomnia in PD recommends the exclusion or otherwise treatment of other sleep disorders, and the identification and treatment of PD specific motor complications and symptoms [18].

In PD patients the amplitude of the rhythmic expression of clock genes such as per 2 as well as the transcription factor bmal 1 was reduced, supposedly influencing gene transcription and inflammatory processes. These changes of circadian processes might be a consequence of the ongoing neurodegenerative process but may also contribute to disease progression itself $[19,20]$. Analyses of a national data banks in Great Britain and Taiwan showed a prodromal incidence risk of insomnia for clinical manifestation of PD of 1,38 (CI $1,11-1,70)$ [21, 22].

In a Finnish questionnaire-based evaluation in 1447 randomly chosen PD patients chronic insomnia was reported by $36,9 \%$ with sleep maintenance problems as the mostly prominent feature in $81 \%$. Others reported a close correlation between low sleep quality, chronic insomnia and restless legs syndrome symptoms [23]. Insomnia in PD seems to be associated with age [24], disease duration, female sex, use of dopamine agonists [25] as well as neuropsychological symptoms such as depression/anxiety, even in de novo PD patients at an early disease stage [26, 27]. In a Dutch longitudinal, prospective, cohort-study insomnia was also associated with motor fluctuations, autonomic dysfunction and hallucinations [28].

Although dopamine agonists were associated with insomnia, extended-release formulations of dopamine agonists seem to improve subjective and objective sleep quality [29] but also motor symptoms [30, 31]. Furthermore, in small case series antidepressants (AD) (i.e. trazodone, doxepine, venlafaxine, nortryptyline), as well as melatonin / ramelteon, pimavanserine and GABAagonists (eszopiclone/zolpidem), exerted a positive effect on insomnia symptoms in PD patients [32-34], whereas duloxetine, quetiapine and clozapine failed to show an improvement in sleep quality [32].

Small interventions studies on CBTi, sometime combined with light therapy, showed a significant improvement in subjective sleep quality [35, 36]. Effects of deep brain stimulation (DBS) on sleep quality are not fully elucidated, yet. While a few questionnaire-based case series on DBS of the subthalamic nuclei resulted in improvement of subjective sleep quality [37-39], a small pilot trial in 5 patients with DBS of the globus pallidus internus failed to show any significant improvement of polysomnographically assessed sleep efficiency and sleep latency as well as subjective sleep quality [40].

Apart from PD, sleep disturbances also occur in other movement disorders. In atypical Parkinson syndromes, such as Multisystem atrophy (MSA), the reported incidence of insomnia is about 70\% compared to $62 \%$ in $\mathrm{PD}$ and also in Progressive supranuclear palsy (PSP) insomnia and disturbed sleep architecture occurred more frequently than in PD (Abbott 2014, [41]). Also, 37,7\% of patients with SCA type 3 suffer from insomnia [42], while a case series of patients from one family with SCA type 2 showed a strong association between insomnia and depression [43].

\section{Recommendations}

- Treating with or switching to extended-release dopaminergics, either as transdermal application or extended-release levodopa, is recommended to improve subjective sleep quality.

- Eszopiclone, doxepine, zolpidem, trazodone, ramelteon and melatonin can be used for the treatment of insomnia in PD patients. However, evidence for the efficacy is insufficient.

- Subjective sleep quality can be improved by treatment with the antipsychotic drug pimavanserine and the antidepressants venlafaxine and nortriptiyline. Treatment with duloxetine, quetiapine and clozapine for insomnia is not recommended due to insufficient evidence of effectiveness.

- Bright light therapy with $1000-7500 \mathrm{~lx}$ for $30-90$ min can be recommended for the treatment of insomnia in PD.

- Optimizing sleep hygiene, CBTi, light therapy and melatonin can be used for the treatment of insomnia in PD

- Consider multifactorial causes of insomnia: comorbidities such as RLS, PLMS, re-emergence of 
motor or non-motor symptoms like akinesia, rigor und/or resting tremor and non-motor symptoms and complications like hallucinations should be identified by adequate diagnostic procedures and addressed specifically.

- Sleep quality of caretakers should also be evaluated since their sleep disorders may lead to impaired quality of care.

\section{Insomnia in multiple sclerosis}

Multiple sclerosis (MS) is frequently associated with sleep disorders: Insomnia (25-55\%), RLS (5-19\%) and sleep related breathing disorder (SRBD, 20-60\%). The causes for insomnia are manifold, and include primary insomnia, pain, nocturia, spasticity and obesity. Some specific MS drug regimens can contribute to insomnia. Insomnia is associated with physical and functional impairment and depression. Depending on criteria and type of assessment $25-54 \%$ of MS patients suffer from nonrestorative sleep [44-46].

At MS onset sleep quality in younger adults is mainly normal [111]. The risk to develop insomnia is associated with increased fatigue scores [47]. A prospective multicenter study in Portugal including 206 MS patients, detected chronic insomnia in $22,6 \%$, frequently associated to female sex, anxiety disorder, fatigue and presence of other comorbidities [48]. Others described a prevalence of insomnia varying between 13,2\% of MS patients with relapsing and remitting MS (RRMS) [49] and 12,5\% with about half of them suffering from RRMS [50]. In 10,000 MS patients and 40,000 matched controls from a database insomnia was identified as a prodromal symptom of MS occurring up to 10 years prior to disease onset [51]. MS patients with insomnia showed a higher arousal level than patients with insomnia only or patients with other neurological disorders [52]. A Dutch study (71 MS patients, 40 controls) showed that in MS patients with sleep disturbances cognitive impairment was pronounced and thalamic functional connectivity was impaired [53].

Data on the effect of disease modifying therapies on sleep and fatigue are scarce. However, some studies describe symptom improvement after or during treatment with disease modifying agents $[47,54]$. On the contrary, in patients with highly active RRMS treated with monthly intramuscular injections of ACTH insomnia was one of the most frequent side effects [55]. However, most studies on insomnia in MS are questionnaire-based and do not take disease severity or drug regimens into account.

A small North American study showed efficacy of CBTi on insomnia symptoms in MS [56], another small study showed decreased melatonin levels after steroid pulse therapy in an MS animal model and in MS patients [57]. Besides, in a case control study with 202 MS patients $5 \mathrm{mg}$ of melatonin exerted positive effects on insomnia symptoms [58].

\section{Recommendations}

- CBTi is recommended despite insufficient data from RCTs.

- Melatonin is recommended despite insufficient data from RCTs.

- ADs are recommended in MS patients with comorbid depression.

- Diagnostic work-up of insomnia is recommended before initiating specific therapies.

- As insomnia often occurs prior to MS onset, MS should be considered in the differential diagnosis of insomnia.

- The association between fatigue and insomnia and the differentiation of both symptoms should be considered in the diagnostic and therapeutic process.

\section{Insomnia in traumatic brain injury}

Traumatic brain injury (TBI) is considered as the most frequent cause for persisting neurological handicap.

More than $50 \%$ of TBI patients suffer from sleep-wake disorders independent of the severity of TBI. Insomnia symptoms exert negative influences on neuroplasticity and the recovery process, as well as on cognition and nocturnal pain sensation and lowers the threshold for psychiatric comorbidity.

A meta-analysis of 1706 participants suffering from TBI of all degrees of severity identified insomnia in $29 \%$, hypersomnia in $28 \%$ and sleep apnea in $25 \%$ of patients [59]. In 95\% of TBI patients decreased CSF-hypocretin-1 levels have been demonstrated during the acute phase. These findings seem to be persistent mainly in patients with a narcolepsy-like phenotype. Congruently, post mortem studies revealed a reduction of hypothalamic hypocretin neurons in TBI patients (Review: [60]). Furthermore, more recent post mortem studies from the same group showed a $41 \%$ reduction of tuberomammilary histaminergic neurons in seven out of 12 patients, but only a $21 \%$ reduction of hypocretin neurons [61]. Female gender, impaired pre-traumatic sleep quality, reduced sleep perception and cognition during the first 2 weeks after acute TBI are predictive for chronic insomnia [62]. Circadian sleep-wake-rhythm disorders, especially the "delayed sleep phase syndrome" are considered as an important differential diagnosis in more than a third of all TBI patients suffering from insomnia [63]. In $42 \%$ of all TBI patients reduced melatonin levels in their evening saliva probes anda delayed nocturnal melatonin synthesis were detected [64]. Patients with severe TBI 
showed an increase of slow wave sleep proportion during their chronic phase, suggesting an increase in posttraumatic neuroplasticity $[63,65]$. No RCTs were identified specifically addressing treatment of insomnia in TBI.

\section{Recommendations}

- Symptom oriented somatic (i.e. CPAP in obstructive sleep apnea) and pharmacological therapy is recommended taking comorbidities into consideration.

- Depending on the clinical conditions the combination of pharmacological therapy and CBTi should be considered.

\section{Insomnia in epilepsy}

The prevalence of epilepsy in adults is about 3\%. In a Chinese study including 150 adult patients with epilepsy (according to the International Classification of Epilepsies and Epileptic Syndrome) suffering from subjective sleep disorders compared to 100 healthy age- and sex matched controls, no differences regarding overall sleep quality using the Pittsburgh Sleep Quality Index (PSQI) were detected. However, the Insomnia Severerity Index (ISI) showed showed significantly higher scores in the epilepsy group. The ISI could identify insomnia in 19\% of all epilepsy patients. Those epilepsy patients with insomnia were significantly more often treated with lamotrigine than those without insomnia [66]. In a prospective longitudinal study comprising 95 patients with unclassified epilepsies, $65,5 \%$ suffered from mild to moderate, and $28,9 \%$ from severe insomnia. Among the predictors for severe insomnia were a history of prior head trauma, previous epilepsy surgery, intake of sedative drugs, and antiepileptic polypharmacotherapy [67].

In a questionnaire-based evaluation comparing sleep quality and insomnia frequency in 208 epilepsy patients and 212 controls, $24 \%$ of epilepsy patients compared to $20 \%$ of controls suffered from chronic sleep initiation difficulties. Chronic insomnia was diagnosed in 36\% of epilepsy patients and 15\% of controls. Sleep quality assessed by the PSQI was classified as impaired in 30\% of epilepsy patients and $16 \%$ in controls [68]. In a Spanish study $22 \%$ of 237 patients with non-refractory epilepsy suffered from sleep disorders compared to $45 \%$ out of 264 patients with refractory epilepsy [69].

In a double-blind, crossover, placebo-controlled study in in 11 children aged $6-11$ years $9 \mathrm{mg}$ slow release melatonin, taken $30 \mathrm{~min}$ prior to bed time, resulted in a significantly reduced sleep latency [70] In 165 American veterans with epilepsy the prevalence of insomnia was $40 \%$. TBI, intake of lamotrigine and affective disorder showed an increased association with insomnia [71]. A non-controlled phase 3 study to evaluate the add-on therapy of brivaracetam on focal epilepsies found insomnia in $3 \%$ of the group treated with $20 \mathrm{mg}$ brivaracetam, and $6,9 \%$ in the group treated with $50 \mathrm{mg}$ [72].

A metaanalysis found a prevalence of $36-74.4 \%$ in adult epilepsy patients (mainly difficulties of maintaining sleep) and $11 \%$ in the pediatric population [73]. In a prospective study in nine patients deep brain stimulation of the anterior thalamic nucleus (ANT) was associated with polysomnographically detected awakenings in $14-67 \%$ of stimulations [74].

Of note, most studies were limited by sleep evaluation using questionnaires instead of polysomnography and epilepsy syndromes were not classified sufficiently.

\section{Recommendations}

- $\mathrm{AE}$ that do not exert influence on sleep should be preferred.

- PSG can be used in patients treated with deep brain stimulation to detect stimulus associated arousals.

- Slow release melatonin can be used in epilepsy to shorten sleep latency (although this has only been verified in children so far).

\section{Insomnia in neuromuscular disease}

A German study compared the frequency of non-motor symptoms in 90 NMD patients with an age matched control group demonstrating significantly more insomnia symptoms in the NMD group [75]. In a Japanese case report insomnia was the leading complaint in a patient with ALS [76].

A Chinese study investigated the use of integrative therapies (chinese herbs, massage, acupuncture) in 258 patients with amyotrophic lateral sclerosis (ALS) and found a higher use of these therapies in ALS patients with insomnia than in those without [77]. An Italian study investigated the association of sleep disorders in facialscapular-humeral dystrophy (FHSD). Polysomnographic data of 32 patients with a genetic form of FSHD were compared to those of an age matched control group. The FSHD group showed longer sleep latencies, shorter sleep duration, more frequent wake episodes and a shorter amount of REM sleep. With disease progression FSHD patients had less body movements in sleep [78].

An Italian and an American study describe each a case with Morvan syndrome. In both cases severe neuromyotonia was combined with severe insomnia, and in both cases antibodies against voltage-gated potassium channels were detected. In one of these cases plasmapheresis resulted in a restitution [79], in another case restitution was achieved by thymectomy [80].

An American study investigated the frequency of sleep disorders using polysomnographic data a in 8 patients 
with myotonic dystrophy type 2 . Five $(62,5 \%)$ patients suffered from insomnia [81].

An Italian study compared the frequency of SRBD in 13 patients with sporadic inclusion body myositis (IBM) to those of age matched controls and found significantly lower sleep efficiency, a higher frequency of nocturnal awakenings, and significantly longer nocturnal wake times [82].

Limitations: Very low patient numbers.

\section{Recommendations}

- Insomnia should be considered as a relevant sleep disorder in ALS.

- Insomnias should be taken into anamnestic and diagnostic considerations in FSHD, IBM and MD2.

- Insomnia must be considered as an integral sleep disorder in patients with Morvan-Syndome, which may improve by treatment with plasmapheresis or thymectomy.

\section{Insomnia in stroke}

Several studies identified insomnia to be responsible for a mild to moderate increase of the risk for stroke, whereas other studies did not detect an increased risk. These discrepancies might partially be attributed to other confounding factors.

The risk for stroke was increased in patients with insomnia in a population based-study in a 10-year followup (HR 1.85; 1.62-2.12) after controlling for other risk factors [83]. A registry-based study with 21,430 patients with insomnia found an increased stroke risk in a fouryear follow-up (HR 1.54; 1.38-1.72) [84]. A populationbased study with 5494 patients found a slightly increased risk for a former stroke in patients with insomnia symptoms (OR 1.26; 1.03-1.55), which could not be confirmed at follow-up over up to 6 years [85]. A metaanalysis of prospective population-based studies found the risk for a later cardio-cerebral vascular event slightly increased in persons with difficulties of sleep initiation (RR 1.27; 1.15-1.40) and people with difficulties to maintain sleep (RR $1.11 ; 1.04-1.19)$ as well as nonrestorative sleep (RR 1.18; 1.05-1.32), whereas the risk for stroke was not elevated in persons reporting early awakenings. The analysis did not discriminate between cardial and cerebral events [86]. Another populationbased study did not find an increased risk for stroke in patients with difficulties initiating and maintaining sleep after controlling for other risk factors. Only in men with either short sleep $(<=5 \mathrm{~h})$ or long sleep duration $(>=10$ h) a slight, but not significant, increase of stroke risk was detected after controlling for several risk factors (HR 1.89; 0.96-3.73 resp.HR 1.72; 0.89-3.33) [87].
The HUNT 3 study $(n=24,715)$ found no increased stroke risk in insomnia patients after correction for confounding variables [88].

\section{Insomnia after stroke}

In a review insomnia was described in $50 \%$ of patients after stroke, of whom 1/3 did not suffer from insomnia prior to stroke. Symptoms are moderated by environmental factors and comorbidities, rarely as a result of brain damage [89]. In a prospective study $59.8 \%$ of patients $(N=214)$ experienced at least one symptom of insomnia in the first month after stroke [90]. In a longitudinal cohort study in $(n=100)$ women impaired sleep quality (as measured by the PSQI) was detected during the acute phase, but not after 6 months of follow-up [91]. A prospective study in 508 patients found symptoms of insomnia 3 months after stroke in $36.6 \%$ patients, in $12.6 \%$ with symptoms sequelae during daytime, mainly in those with stroke of the frontal lobe [92].

A multicentric, prospective study $(n=809)$ found insomnia according to DSM IV criteria in $26.9 \%$ of all patients and any type of sleep disorder in 56.7\% [93]. A prospective multicenter study with 441 patients, age $<65$ years found insomnia in $30-37 \%$ of all patients after 1 year of follow-up. 16\% suffered from chronic insomnia after 6 months, and had an increased risk for feeling depressed (OR 6.75; 2.78-16.4), anxious (OR 3.31; 1.54-7.09) or to have a physical handicap (OR 3.60; 2.07-6.25) [94].

Quality of life in the first weeks after stroke was worse in patients with insomnia than in those without [95]. Health related quality of life HRQol was reduced in 336 patients with insomnia which occurred after stroke [96]. Symptoms of insomnia and reduced HRQol with physical and even more mental factors correlated in 214 patients 1 month after stroke [90].

Treatment of insomnia after stroke should comprise behavioral strategies such as a silent environment during the night with protection from too much light, during daytime much light exposure and physical activity. Drug treatment should comprise benzodiazepine receptoragonists (zolpidem) or sedating antidepressants (amitriptyline, trazodone, mianserin, mirtazapine) [89]. A randomized controlled pilot-study in 15 patients found improved sleep disorder after 4 months of CBTi compared to unspecific treatment, which did not persist at follow-up [97]. A small prospective study in 30 patients with subacute stroke did not show differences of function, cognition and depression between stroke patients after stroke and patients with stroke and insomnia treated with hypnotics ( $n=15$ Zolpidem, in one patient additional 0.125 $\mathrm{mg}$ triazolam and in two patients trazodone $25 \mathrm{mg}$ ) after 3 weeks. This result indicates that hypnotics do not hamper rehabilitation [98]. Acupuncture has proven to be helpful in the treatment of insomnia after stroke [99]. 


\section{Recommendations}

- Since insomnia occurs frequently in acute stroke, screening for insomnia should be performed.

- Insomnia in stroke patients should be treated to improve the outcome of stroke and to improve quality of life.

- Therapies using bright light, benzodiazepinereceptor agonists, sedating antidepressants, CBTi and acupuncture can be recommended.

\section{Insomnia in dementia and prion-diseases}

Literature of the past 20 years has given proof that sleep disorders of any kind may be one of the first signs or may even directly contribute to the development of dementias, with insomnia being of special interest for the development of Alzheimer dementia (AD). Early diagnosis and treatment of sleep disorders dementia could add to the prevention of dementias.

Aggregation of beta amyloid (Aß) starts about 20 years prior to diagnosis of AD. Short sleep duration seems to impair $A ß$ elimination by the glymphatic system and seems to enhance production of $A ß$, leading to $A ß$ accumulation in the brain.

A population-based Italian study found dementia in 86 of 750 persons $>65$ years. Of those $84,7 \%$ had insomnia, $26,2 \%$ snoring and apneas, $25.7 \%$ leg movements, $30,6 \%$ excessive daytime sleepiness (eds)). EDS was the only predictor for cognitive decline [100].

In a crosssectional questionnaire-based evaluation in 500 patients with early forms of Lewy body dementia (DLB), Alzheimer (AD) and PD dementia (PDD) detected insomnia in $29,9 \%$, nocturnal cramps in $24,1 \%$, eds in $22,6 \%$, RLS in $20,7 \%$ and RBD in $18,5 \%$ of patients. Patients with $\mathrm{AD}$ reported lower frequencies of sleep disturbances compared to DLB and PDD [101].

Patients suffering from long lasting insomnia aged 5065 years have a higher risk to develop dementia (HR, 5.22 ; 95\% CI, 2.62-10.41) than patients $>65$ years without insomnia. Use of hypnotics with long half-life and high doses of hypnotics increase the risk for dementia [102]. In persons $>75$ years difficulties to maintain sleep increased the risk for cognitive decline of previously cognitively not impaired persons [103].

Sleep disorders with prolonged sleep latency and low sleep efficiency were associated with cognitive frontal executive and visuospatial dysfunctions [104].

Polysomnographies of patients with Creutzfeldt-Jakob disease showed changes of the sleep structure: Reduction of total sleep time, sleep spindles and $\mathrm{K}$ complexes as well as sleep fragmentation and REM sleep without atonia. Comorbid sleep disorders in these patients are hypersomnia in $39 \%$, insomnia in $46 \%$, RLS in $25 \%$ and parasomnias in $32 \%$. Patients can benefit from treatment of their comorbid sleep disorders [105].

A combination of bright light exposition and walking exercise for $>4$ days/week increased sleep duration measured by actigraphy in AD patients [106].

In an evidence-based guideline for " Prescribing antipsychotics for behavioural and psychological symptoms of dementia and insomnia" (VSPD) recommendations on the handling of insomnia symptoms are given: 1 . in patients, in which symptoms are stabilized or who show no response to adequate treatment for 3 months, antipsychotics should be slowly tapered every 1-2 weeks in collaboration with the patient and the caregiver (strong recommendation, moderate evidence). This recommendation is not applicable to psychotic patients 2. For adults with primary insomnia treated for any duration or secondary insomnia, in which underlying comorbidities are managed, antipsychotics can be stopped; tapering is not needed (good practice recommendation) [107].

A Cochrane meta-analysis (2013) including 10 studies showed little or no evidence, that tapering of neuroleptics that had been administered over a long time period in patients with VSPD older than 65 years had any effect on the symptoms [108].

A 2016 Cochrane analysis [109] on four RCTs with melatonin administration of up to $10 \mathrm{mg}$ and slow release melatonin of $2.5 \mathrm{mg}$ over $8-10$ weeks in patients with dementia and sleep disorders did not show significant improvement for any of the sleep parameters. In 30 patients treated with trazodone sleep duration and sleep efficiency increased, but there was no improvement in nocturnal wake time and frequency of awakenings. Trazodone $8 \mathrm{mg}$ administered over 8 weeks did not show any effect on sleep parameters.

Limitations: Most studies assessed sleep disorders with sleep questionnaires, interviews or actigraphy. Few studies are RCTs. All of the studies used different protocols over different time periods. The recommendations are therefore of very low evidence and do not imply that certain treatments may not be helpful or efficacious.

\section{Recommendations}

- In patients with behavioral problems caused by dementia, whose insomnia problems have been treated insufficiently with neuroleptics over years, neuroleptics should be tapered.

- No benefit for the treatment of insomnia in demented patients could be shown for hypnotics such as benzodiazepines, benzodiazepine receptor agonists and phytotherapy. These drugs should not be administered in demented patients with insomnia in general. 
- Immediate release, slow release melatonin and melatonin-agonists may be options in the treatment of insomnia patients with AD.

- Trazodone $50 \mathrm{mg}$ at night can be recommended since it improves sleep duration and sleep onset time in patients with $\mathrm{AD}$, but there is no evidence, that it improves sleep fragmentation.

- Bright light therapy (2500 Lux full spectrum) in combination with $30 \mathrm{~min}$ of walking on at least 4 days per week can be recommended to improve sleep duration.

\section{Financing}

The travelling cost of participants were covered by DGN. The guideline was not sponsored.

\section{Methods of guideline development \\ Participants from medical societies}

For the German Society for Psychiatry and Psychotherapy DGPPN and for the German Society for Sleep Medicine: Prof. Dr. rer. Soc. Riemann.

For the German Society of Neurologists DGN and for the German Society for Clinical Neurophysiology and Functional Imaging DGKN Prof. Dr. med. Mayer.

German Alzheimer Society (Deutsche Alzheimer Gesellschaft e.V., Selbsthilfe Demenz): Mrs. S. Jansen, Berlin.

German Multiple Sclerosis Society (DMSG Bundesverband): Dr. D. Pöhlau, Aasbach.

German Parkinson Society (Deutsche Parkinson Gesellschaft e.V.): Dr. med. Wiebke Hermann, Rostock.

German Epilepsy Union (Deutsche Epilepsievereinigung): Participation was not possible.

Germain Migraine and Headache Society (Deutsche Migräne- und Kopfschmerzgesellschaft e.V.): Prof. Dr. med. Stephan Evers, Coppenbrügge.

German Stroke Society (Deutsche Schlaganfallgesellschaft): participation was not considered to be necessary.

\section{Participation of patient groups}

Deutsche Alzheimer Gesellschaft e.V., Selbsthilfe Demenz, Friedrichstr. 236, 10,969 Berlin.

Deutsche Multiple Sklerose Gesellschaft, Bundesverband e.v. DMSG, Krausestr. 50, 30,171 Hannover.

Deutsche Parkinson Vereinigung - Bundesverband e.V., Moselstraße 31, 41,464 Neuss.

Deutsche Epilepsievereinigung e.V., Bundesgeschäftsstelle, Zillestraße 102, 10,585 Berlin.

Deutsche Migräne- und Kopfschmerzgesellschaft, Migräne- und Kopfschmerzklinik Königstein; Ölmühlweg 31, 61,462 Königstein im Taunus.

\section{Search and selection of scientific literature}

Search used medline, pubmed, embase, web of science und Cochrane database. Search included years 11/2009$12 / 2018$ and was restricted to adult population. Search terms were "insomnia in neurologic disease", "insomnia in central neurologic diseases”, and "insomnia and - respective neurological diseases").

Articles had to contain at least one definition of insomnia according to recent classification systems. All randomized, controlled studies with $>5$ patients were included. If studies with high evidence were lacking, studies of lower evidence were included, if they met the selection criteria. Evidence classification (class $1-! \mathrm{V}$ ) was perfomed according to standardized European Procedures [110]. Levels of recommendation are A-D. Literature was categorized independantly by two experts according to Oxford Centre for Evidence-based Medicine Levels of Evidence (2001).

\section{Consensus methods}

Modified Delphi procedure: PD Dr. Sitter.

Consensus was reached with a nominal group process and delphi technique in two consensus conferences.

\section{Appendix}

Links to sleep questionnaires

Pittsburg Sleep Quality Index https://www. outcometracker.org/library/PSQI.pdf

SF-A: https://www.researchgate.net/publication/2 85321057_Schlaffragebogen_A_und_B

Abend Morgen Protokoll: https://www.dgsm.de/ downloads/fachinformationen/frageboegen/2wochen.pdf

Insomnia Severiy Index: https://deploymentpsych.org/ system/files/member_resource/Insomnia\%20Severity\%2 0Index\%20-ISI.pdf

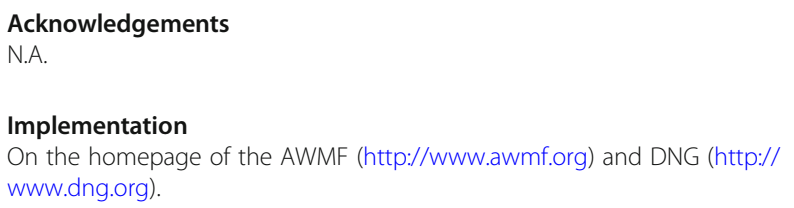

Authors' contributions

All authors wrote one or two chapters. The authors read and approved the final manuscript.

\section{Funding}

None.

Availability of data and materials

N.A.

Ethics approval and consent to participate N.A.

Consent for publication

All authors and participating patient groups gave their consent. 


\section{Competing interests}

None.

\section{Author details}

${ }^{1}$ Neurologische Abteilung der Hephata-Klinik, Schimmelpfengstrasse 6, 34613 Schwalmstadt-Treysa, Germany. ${ }^{2}$ Neurologische Abteilung der Philipps-Universität Marburg, Mamburg, Germany. ${ }^{3}$ Klinik Maria Frieden, Klinik für Neurologie, Am Krankenhaus 1, 48291 Telgte, Germany. ${ }^{4}$ Krankenhaus Lindenbrunn, Abteilung Neurologie, Lindenbrunn 1, 31863 Coppenbrügge, Germany. ${ }^{5}$ Klinik und Poliklinik für Neurologie und Deutsches Zentrum für Neurodegenerative Erkrankungen e.V. (DZNE), Gehlsheimer Str. 20, 18147 Rostock, Germany. ${ }^{6}$ Deutsche Alzheimer Gesellschaft e.V. Selbsthilfe Demenz, Friedrichstr. 236, 10969 Berlin, Germany. ${ }^{7}$ Klin. Schlaf- und Neuroimmunologie, Private Universität Witten/Herdecke gGmbH, Alfred-Herrhausen-Str. 50, 58448 Witten, Germany. ${ }^{8}$ Paracelsus Elena Klinik, Schanzenstr. 85 Dr. med Dieter Pöhlau, 34130 Kassel, Germany. ${ }^{9}$ DRK Kamillus Klinik, Hospitalstr. 6, 53567 Asbach, Germany. ${ }^{10}$ Psychiatrische Universitätsklinik Freiburg, Hauptstraße 5, 79104 Freiburg, Germany. ${ }^{11}$ LKH Graz II, Standort Süd, Wagner Jauregg Platz 1, A-8053 Graz, Austria.

${ }^{12}$ Universitätsklinik für Psychiatrie und Psychotherapie, Murtenstrasse 21, 3008 Bern, Switzerland. ${ }^{13}$ Paracelsus-Elena-Klinik, Klinikstr. 16, 34128 Kassel, Germany. ${ }^{14}$ Neurologische Klinik Reithofpark, Reithof 1, 83075 Bad Feilnbach, Germany.

Received: 10 January 2021 Accepted: 12 January 2021

Published online: 10 March 2021

\section{References}

1. WHO (1992). Kapitel V-F51.0 Insomnien. In ICD-10 (international statistical classification of diseases and related health problems). Genf: WHO.

2. American Psychiatric Association (Hrsg) (2013). DSM5 (diagnostic and statistical manual of mental disorders). Washington DC: American Psychiatric Association.

3. American Academy of Sleep Medicine (Hrsg) (2014). ICSD-3 (international classification of sleep). Darien: American Association of Sleep Medicine.

4. American Acadamy of Sleep Medicine (2014). International Classification of Sleep Disorders - Third Edition (ICSD-3). Rochester: American Sleep Disorders Association.

5. Headache Classification Committee of the International Headache Society (IHS) (2018). The international classification of headache disorders, 3rd edition. Cephalalgia, 38, 1-211.

6. Kim, J., Cho, S. J., Kim, W. J., Yang, K. I., Yun, C. H., \& Chu, M. K. (2017). Insufficient sleep is prevalent among migraineurs: A population-based study. The Journal of Headache and Pain, 18, 50.

7. Kim, J., Cho, S. J., Kim, W. J., Yang, K. I., Yun, C. H., \& Chu, M. K. (2017). Insomnia in tension-type headache: A population-based study. The Journal of Headache and Pain, 18, 95.

8. Song, T. J., Yun, C. H., Cho, S. J., Kim, W. J., Yang, K. I., \& Chu, M. K. (2018). Short sleep duration and poor sleep quality among migraineurs: $\mathrm{A}$ population-based study. Cephalalgia, 38, 855-864.

9. Verma, R., Nagar, K. K., Garg, R. K., Uniyal, R., Sharma, P. K., \& Pandey, S. (2016). Study of sleep disorders and polysomnographic evaluation among primary chronic daily headache patients. Journal of Neurosciences in Rural Practice, 7(Suppl 1), S72-S75.

10. Engstrøm, M., Hagen, K., Bjørk, M., Stovner, L. J., Stjern, M., \& Sand, T. (2014). Sleep quality, arousal and pain thresholds in tension-type headache: A blinded controlled polysomnographic study. Cephalalgia, 34, 455-463.

11. Shand, B., Goicochea, M. T., Valenzuela, R., Fadic, R., Jensen, R., Tassorelli, C., ... the COMOESTAS CONSORTIUM (2015). Clinical and demographical characteristics of patients with medication overuse headache in Argentina and Chile: Analysis of the Latin American Section of COMOESTAS Project. The Journal of Headache and Pain, 16, 83.

12. Smitherman, T. A., Walters, A. B., Davis, R. E., Ambrose, C. E., Roland, M. Houle, T. T., \& Rains, J. C. (2016). Randomized controlled pilot trial of behavioral insomnia treatment for chronic migraine with comorbid insomnia. Headache, 56, 276-291.

13. Spierings, E. L., McAllister, P. J., \& Bilchik, T. R. (2015). Efficacy of treatment of insomnia in migraineurs with eszopiclone (Lunesta ${ }^{\oplus}$ ) and its effect on total sleep time, headache frequency, and daytime functioning: A randomized, double-blind, placebo-controlled, parallelgroup, pilot study. Cranio, 33, 115-121.
14. Stavitsky, K., Neargarder, S., Bogdanova, Y., McNamara, P., \& Cronin-Golomb, A. (2012). The impact of sleep quality on cognitive functioning in Parkinson's disease. Journal of the International Neuropsychological Society, 18(1), 108-117.

15. Videnovic, A. (2017). Management of sleep disorders in Parkinson's disease and multiple system atrophy. Movement Disorders, 32(5), 659-668.

16. Högl, B., Arnulf, I., Comella, C., Ferreira, J., Iranzo, A., Tilley, B., ... Goetz, C. G. (2010). Scales to assess sleep impairment in Parkinson's disease: Critique and recommendations. Movement Disorders, 25, 2704-2716.

17. Seppi, K., Weintraub, D., Coelho, M., Perez-Lloret, S., Fox, S. H., Katzenschlager, R., ... Sampaio, C. (2011). The Movement Disorder Society evidence-based medicine review update: Treatment of the non-motor symptoms of Parkinson's disease. Movement Disorders, 26(S3), 42.

18. Ferreira, J. J., Katzenschlager, R., Bloem, B. R., Bonuccelli, U., Burn, D., Deuschl, G., ... Oertel, W. H. (2013). Summary of the recommendations of the EFNS/ MDS-ES review on therapeutic management of Parkinson's disease. European Journal of Neurology, 20, 5-15.

19. Abbott, S. M., \& Videnovic, A. (2016). Chronic sleep disturbance and neural injury: Links to neurodegenerative disease. Nature and Science of Sleep, 8 , $55-61$.

20. Hood, S., \& Amir, S. (2017). Neurodegeneration and the circadian clock. Frontiers in Aging Neuroscience, 9, 170.

21. Hsiao, Y. H., Chen, Y. T., Tseng, C. M., Wu, L. A., Perng, D. W., Chen, Y. M., ... Chou, K. T. (2017). Sleep disorders and an increased risk of Parkinson's disease in individuals with non-apnea sleep disorders: A population-based cohort study. Journal of Sleep Research, 26, 623-628.

22. Schrag, A., Horsfall, L., WIters, K., Noyce, A., \& Petersen, I. (2015). Prediagnostic presentations of Parkinson's disease in primary care: A casecontrol study. Lancet Neurology, 14, 57-64.

23. Ylikoski, A., Martikainen, K., Sieminski, M., \& Partinen, M. (2015). Parkinson's disease and insomnia. Neurological Sciences, 36, 2003-2010.

24. Mahale, R., Yadav, R., \& Pal, P. K. (2015). Quality of sleep in young onset Parkinson's disease: Any difference from older onset Parkinson's disease. Parkinsonism \& Related Disorders, 21, 461-464.

25. Telarovic, S., Mijatovic, D., \& Telarovic, I. (2015). Effects of various factors on sleep disorders and quality of life in Parkinson's disease. Acta Neurologica Belgica, 115, 615-621.

26. Rutten, S., Vriend, C., van der Werf, Y. D., Berendse, H. W., Weintraub, D., \& van den Heuvel, O. A. (2017). The bidirectional longitudinal relationshiip between insomnia, depression and anxiety in patients with early-stage, medication-naive Parkinson's disease. Parkinsonism \& Related Disorders, 39, $31-36$.

27. Tholfsen, L. K., Larsen, J. P., Schulz, J., Tysnes, O. B., \& Gjerstad, M. D. (2017). Changes in insomnia subtypes in early Parkinson's disease. Neurology, 88, 352-358.

28. Zhu, K., van Hilten, J. J., \& Marinus, J. (2016). The course of insomnia in Parkinson's disease. Parkinsonism \& Related Disorders, 33, 51-57.

29. Ferreira, T., Prabhakar, S., \& Kharbanda, P. S. (2014). Sleep disturbances in drug naive Parkinson's disease (PD) patients and effect of levodopa on sleep. Ann Indian Acad Neurol, 17, 416-419.

30. Pahwa, R., Stacy, M. A., Factor, S. A., et al. (2007). Ropinirole 24-hour prolonged release: Randomized, controlled study in advanced Parkinson disease. Neurology, 68, 1108-1115.

31. Trenkwalder, C., Kies, B., Rudzinska, M., et al. (2011). Rotigotine effects on early morning motor function and sleep in Parkinson's disease: A doubleblind, randomized, placebo-controlled study (RECOVER). Movement Disorders, 26, 90-99.

32. Amara, A. W., Chahine, L. M., \& Videnovic, A. (2017). Treatment of sleep dysfunction in Parkinson's disease. Current Treatment Options in Neurology, 19, 26.

33. Rodrigues, T. M., Castro Caldas, A., \& Ferreira, J. J. (2016). Pharmacological interventions for daytime sleepiness and sleep disorders in Parkinson's disease: Systematic review and meta-analysis. Parkinsonism \& Related Disorders, 27, 25-34.

34. Trotti, L. M., \& Karroum, E. G. (2016). Melatonin for sleep disorders in patients with neurodegenerative diseases. Current Neurology and Neuroscience Reports, 16, 63.

35. Rios Romenets, S., Creti, L., Fichten, C., et al. (2013). Doxepin and cognitive behavioural therapy for insomnia in patients with Parkinson's disease - A randomized study. Parkinsonism \& Related Disorders, 19, 670-675. 
36. Yang, H., \& Petrini, M. (2012). Effect of cognitive behavior therapy on sleep disorder in Parkinson's disease in China: A pilot study. Nursing \& Health Sciences, 14, 458-463.

37. Amara, A. W., Standaert, D. G., Guthrie, S., Cutter, G., Watts, R. L., \& Walker, H. C. (2012). Unilateral subthalamic nucleus deep brain stimulation improves sleep quality in Parkinson's disease. Parkinsonism \& Related Disorders, 18, 63-68.

38. Chahine, L. M., Ahmed, A., \& Sun, Z. (2011). Effects of STN DBS for Parkinson's disease on restless legs syndrome and other sleep-related measures. Parkinsonism \& Related Disorders, 17, 208-211.

39. Choi, J. H., Kim, H. J., Lee, J. Y., Yoo, D., Im, J. H., Paek, S. H., \& Jeon, B. (2019). Long-term effects of bilateral subthalamic nucleus stimulation on sleep in patients with Parkinson's disease. PLoS One, 14(8).

40. Tolleson, C. M., Bagai, K., Walters, A. S., \& Davis, T. L. (2016). A pilot study assessing the effects of pallidal deep brain stimulation on sleep quality and polysomnography in Parkinson's patients. Neuromodulation, 19, 724-730.

41. Walsh, C. M., Ruoff, L., Walker, K., Emery, A., Varbel, J., Karageorgiou, E., Luong, P. N., Mance, I., Heuer, H. W., Boxer, A. L., Grinberg, L. T., Kramer, J. H., Miller, B. L., \& Neylan, T. C. (2017). Sleepless Night and Day, the Plight of Progressive Supranuclear Palsy. Sleep. 40(11), zsx154. https://doi.org/10.1093/ sleep/zsx154

42. Pedroso, J. L., Braga-Neto, P., Martinez, C. R. J., Rezende Filho, F. M., SobreiraNeto, M. A., Prado, L. B., ... Barsottini, O. G. (2016). Sleep disorders in Machado-Joseph disease. Current Opinion in Psychiatry, 29, 402-408.

43. Hsu, C. H., Chen, Y. L., Pei, D., Yu, S. M., \& Liu, I. C. (2016). Depression as the primary cause of insomnia and excessive daytime sleepiness in a family with multiple cases of spinocerebellar ataxia. Journal of Clinical Sleep Medicine, 17, 1059-1061.

44. Veauthier, C. (2015). Sleep disorders in multiple sclerosis. Review. Current Neurology and Neuroscience Reports, 15, 21

45. Braley, T. J., \& Boudreau, E. A. (2016). Sleep disorders in multiple sclerosis. Current Neurology and Neuroscience Reports, 16, 50.

46. Caminero, A., \& Bartolomé, M. (2011). Sleep disturbances in multiple sclerosis. Journal of the Neurological Sciences, 309, 86-91.

47. Kotterba, S., Neusser, T., Norenberg, C., et al. (2018). Sleep quality, daytime sleepiness, fatigue, and quality of life in patients with multiple sclerosis treated with interferon beta-1b: results from a prospective observational cohort study. BMC Neurology, 18, 123.

48. Viana, P., Rodrigues, E., Fernandes, C., et al. (2015). InMS: Chronic insomnia disorder in multiple sclerosis - a Portuguese multicentre study on prevalence, subtypes, associated factors and impact on quality of life. Multiple Sclerosis and Related Disorders, 4, 477-483.

49. Brozek, P., Brachmanska, M., Rabiczko, K., Bulska, W., Ciulkowicz, M., \& Krzystanek, E. (2017). Depression, sleep disturbances and anxiety in patients with relapsing-remitting multiple sclerosis: A longitudinal cohort observation. Psychiatria Danubina, 29(S3), 464-468.

50. González-Platas, M., González-Platas, J., Bermúdez-Hernández, M., PérezMartín, M. Y., Croissier-Elías, C., \& Pérez-Lorensu, P. J. (2016). Low prevalence of sleep disorders in demyelinating disease in a northern Tenerife population. Journal of Clinical Sleep Medicine, 12, 805-811.

51. Disanto, G., Zecca, C., MacLachlan, S., et al. (2018). Prodromal symptoms of multiple sclerosis in primary care. Annals of Neurology, 83(6), 1162-1173.

52. Schellaert, V., Labauge, P., Lebrun, C., et al. (2018). Psychological processes associated with insomnia in patients with multiple sclerosis. Sleep, 41.

53. van Geest, Q., Westerik, B., van der Werf, Y. D., Geurts, J. J., \& Hulst, H. E. (2017). The role of sleep on cognition and functional connectivity in patients with multiple sclerosis. Journal of Neurology, 264, 72-80.

54. Penner, I. K., et al. (2015). Improvement in fatigue during natalizumab treatment is linked to improvement in depression and day-time sleepiness. Frontiers in Neurology, 6, 18

55. Berkovich, R., Bakshi, R., Amezcua, L., et al. (2017). Adrenocorticotropic hormone versus methylprednisolone added to interferon $\beta$ in patients with multiple sclerosis experiencing breakthrough disease: A randomized, raterblinded trial. Therapeutic Advances in Neurological Disorders, 10, 3-17.

56. Clancy, M., Drerup, M., \& Sullivan, A. B. (2015). Outcomes of cognitivebehavioral treatment for insomnia on insomnia, depression, and fatigue for individuals with multiple sclerosis: A case series. International Journal of MS Care, 17, 261-267.

57. Dokoohaki, S., Ghareghani, M., Ghanbari, A., Farhadi, N., Zibara, K., \& Sadeghi, H. (2017). Corticosteroid therapy exacerbates the reduction of melatonin in multiple sclerosis. Steroids, 128, 32-36
58. Adamczyk-Sowa, M., Pierzchala, K., Sowa, P., Mucha, S., Sadowska-Bartosz, I. Adamczyk, J., \& Hartel, M. (2014). Melatonin acts as antioxidant and improves sleep in MS patients. Neurochemical Research, 39, 1585-1593.

59. Mathias, J. L., \& Alvaro, P. K. (2012). Prevalence of sleep disturbances, disorders, and problems following traumatic brain injury: A meta-analysis. Sleep Medicine, 13(7), 898-905.

60. Baumann, C. R. (2016). Sleep and traumatic brain injury. Sleep Medicine Clinics, 11(1), 19-23.

61. Valko, P. O., Gavrilov, Y. V., Yamamoto, M., et al. (2016). Damage to arousalpromoting brainstem neurons with traumatic brain injury. Sleep, 39(6), 1249-1252.

62. Theadom, A., Cropley, M., Parmar, P., et al. (2015). Sleep difficulties one year following mild traumatic brain injury in a population-based study. Sleep Medicine, 16(8), 926-932.

63. Sandsmark, D, K., Elliott, J, E., \& Lim, M. M. (2017). Sleep-Wake Disturbances After Traumatic Brain Injury: Synthesis of Human and Animal Studies. Sleep. 40(5), zsx044. https://doi.org/10.1093/sleep/zsx044

64. Grima, N. A., Ponsford, J. L., Hilaire, S., et al. (2016). Circadian melatonin rhythm following traumatic brain injury. Neurorehabilitation and Neural Repair, 30(10), 972-977.

65. Imbach, L. L., Valko, P. O., Li, T., Maric, A., et al. (2015). Increased sleep need and daytime sleepiness 6 month safter traumatic brain injury: A prospective controlled clinical trial. Brain, 138(Pt 3), 726-735.

66. Shen, Y., Zhang, M., Wang, Y., Wang, L., Xu, X., Xiao, G., et al. (2017). Subjective sleep disturbance in Chinese adults with epilepsy: Associations with affective symptoms. Epilepsy Research, 135, 150-157.

67. Yang, K. I., Grigg-Damberger, M., Andrews, N., O'Rourke, C., Bena, J., \& Foldvary-Schaefer, N. (2016). Severity of self-reported insomnia in adults with epilepsy is related to comorbid medical disorders and depressive symptoms. Epilepsy \& Behavior, 60, 27-32.

68. Ismayilova, V., Demir, A. U., \& Tezer, F. I. (2015). Subjective sleep disturbance in epilepsy patients at an outpatient clinic: A questionnaire-based study on prevalence. Epilepsy Research, 115, 119-125.

69. Garcia-Morales, I., Gil-Nagel, A., de Rosendo, J., \& Torres-Falcon, A. (2014). Sleep disorders and quality of life in refractory partial epilepsy: Results of the SLEEP study. Revista de Neurologia, 58(4), 152-160.

70. Jain, S. V., Horn, P. S., Simakajornboon, N., Beebe, D. W., Holland, K., Byars, A. W., et al. (2015). Melatonin improves sleep in children with epilepsy: A randomized, double-blind, crossover study. Sleep Medicine, 16(5), 637-644.

71. Lopez, M. R., Cheng, J. Y., Kanner, A. M., Carvalho, D. Z., Diamond, J. A., \& Wallace, D. M. (2013). Insomnia symptoms in South Florida military veterans with epilepsy. Epilepsy \& Behavior, 27(1), 159-164.

72. Biton, V., Berkovic, S. F., Abou-Khalil, B., Sperling, M. R., Johnson, M. E., \& Lu, S. (2014). Brivaracetam as adjunctive treatment for uncontrolled partial epilepsy in adults: A phase III randomized, double-blind, placebo-controlled trial. Epilepsia, 55(1), 57-66.

73. Macedo, P., Oliveira, P. S., Foldvary-Schaefer, N., \& Gomes, M. D. M. (2017). Insomnia in people with epilepsy: A review of insomnia prevalence, risk factors and associations with epilepsy-related factors. Epilepsy Research, 135, 158-167.

74. Voges, B. R., Schmitt, F. C., Hamel, W., House, P. M., Kluge, C., Moll, C. K., et al. (2015). Deep brain stimulation of anterior nucleus thalami disrupts sleep in epilepsy patients. Epilepsia, 56(8), e99-e103.

75. Gunther, R., Richter, N., Sauerbier, A., Chaudhuri, K. R., Martinez-Martin, P., Storch, A., et al. (2016). Non-motor symptoms in patients suffering from motor neuron diseases. Frontiers in Neurology, 7, 117.

76. Takekawa, H., Kubo, J., Miyamoto, T., Miyamoto, M., \& Hirata, K. (2001). Amyotrophic lateral sclerosis associated with insomnia and the aggravation of sleep-disordered breathing. Psychiatry and Clinical Neurosciences, 55(3), 263-264.

77. Pan, W., Chen, X., Bao, J., Bai, Y., Lu, H., Wang, Q., et al. (2013). The use of integrative therapies in patients with amyotrophic lateral sclerosis in shanghai, China. Evidence-based Complementary and Alternative Medicine, 2013, 613596

78. Della Marca, G., Frusciante, R., Dittoni, S., Vollono, C., Losurdo, A., Testani, E et al. (2010). Decreased nocturnal movements in patients with facioscapulohumeral muscular dystrophy. Journal of Clinical Sleep Medicine, 6(3), 276-280

79. Provini, F., Marconi, S., Amadori, M., Guaraldi, P., Pierangeli, G., Cortelli, P., et al. (2011). Morvan chorea and agrypnia excitata: When videopolysomnographic recording guides the diagnosis. Sleep Medicine, 12(10), 1041-1043. 
80. Abou-Zeid, E., Boursoulian, L. J., Metzer, W. S., \& Gundogdu, B. (2012). Morvan syndrome: A case report and review of the literature. Journal of Clinical Neuromuscular Disease, 13(4), 214-227.

81. Shepard, P., Lam, E. M., St Louis, E. K., \& Dominik, J. (2012). Sleep disturbances in myotonic dystrophy type 2. European Neurology, 68(6), 377380.

82. Della Marca, G., Sancricca, C., Losurdo, A., Di Blasi, C., De Fino, C., Morosetti, R., et al. (2013). Sleep disordered breathing in a cohort of patients with sporadic inclusion body myositis. Clinical Neurophysiology, 124(8), 16151621.

83. Hsu, C. Y., Chen, Y. T., Chen, M. H., Huang, C. C., Chiang, C. H., Huang, P. H., ... Chan, W. L. (2015). The association between insomnia and increased future cardiovascular events: A Nationwide population-based study. Psychosomatic Medicine, 77(7), 743-751.

84. Wu, M. P., Lin, H. J., Weng, S. F., Ho, C. H., Wang, J. J., \& Hsu, Y. W. (2014). Insomnia subtypes and the subsequent risks of stroke: Report from a nationally representative cohort. Stroke, 45(5), 1349-1354.

85. Jaussent, I., Empana, J. P., Ancelin, M. L., Besset, A., Helmer, C., Tzourio, C., .. Dauvilliers, Y. (2013). Insomnia, daytime sleepiness and cardiocerebrovascular diseases in the elderly: A 6-year prospective study. PLoS One, 8(2), e56048.

86. He, Q., Zhang, P., Li, G., Dai, H., \& Shi, J. (2017). The association between insomnia symptoms and risk of cardio-cerebral vascular events: A metaanalysis of prospective cohort studies. European Journal of Preventive Cardiology, 24(10), 1071-1082.

87. Helbig, A. K., Stockl, D., Heier, M., Ladwig, K. H., \& Meisinger, C. (2015). Symptoms of insomnia and sleep duration and their association with incident strokes: Findings from the population-based MONICA/KORA Augsburg cohort study. PLoS One, 10(7), e0134480.

88. Sivertsen, B., Lallukka, T., Salo, P., Pallesen, S., Hysing, M., Krokstad, S., \& Simon, O. (2014). Insomnia as a risk factor for ill health: Results from the large population-based prospective HUNT study in Norway. Journal of Sleep Research, 23(2), 124-132.

89. Hermann, D. M., \& Bassetti, C. L. (2016). Role of sleep-disordered breathing and sleep-wake disturbances for stroke and stroke recovery. Neurology, 87(13), 1407-1416

90. Kim, W. H., Jung, H. Y., Choi, H. Y., Park, C. H., Kim, E. S., Lee, S. J., ... Joa, K. L. (2017). The associations between insomnia and health-related quality of life in rehabilitation units at 1 month after stroke. Journal of Psychosomatic Research, 96, 10-14.

91. Bakken, L. N., Kim, H. S., Finset, A., \& Lerdal, A. (2014). Subjective sleep quality in relation to objective sleep estimates: Comparison, gender differences and changes between the acute phase and the six-month follow-up after stroke. Journal of Advanced Nursing, 70(3), 639-650.

92. Chen, Y. K., Lu, J. Y., Mok, V. C., Ungvari, G. S., Chu, W. C., Wong, K. S., \& Tang, W. K. (2011). Clinical and radiologic correlates of insomnia symptoms in ischemic stroke patients. International Journal of Geriatric Psychiatry, 26(5), 451-457.

93. Joa, K. L., Kim, W. H., Choi, H. Y., Park, C. H., Kim, E. S., Lee, S. J., ... Jung, H. Y. (2017). The effect of sleep disturbances on the functional recovery of rehabilitation inpatients following mild and moderate stroke. American Journal of Physical Medicine \& Rehabilitation, 96(10), 734-740.

94. Glozier, N., Moullaali, T. J., Sivertsen, B., Kim, D., Mead, G., Jan, S., ... Hackett, M. L. (2017). The course and impact of Poststroke insomnia in stroke survivors aged 18 to 65 years: Results from the psychosocial outcomes in StrokE (POISE) study. Cerebrovascular Diseases Extra, 7(1), 9-20.

95. Kim, K. T., Moon, H. J., Yang, J. G., Sohn, S. I., Hong, J. H., \& Cho, Y. W. (2017). The prevalence and clinical significance of sleep disorders in acute ischemic stroke patients-a questionnaire study. Sleep \& Breathing, 21(3), 759-765.

96. Tang, W. K., Grace Lau, C., Mok, V., Ungvari, G. S., \& Wong, K. S. (2015). Insomnia and health-related quality of life in stroke. Topics in Stroke Rehabilitation, 22(3), 201-207.

97. Nguyen, S., Wong, D., McKay, A., Rajaratnam, S. M. W., Spitz, G., Williams, G., Mansfield, D., \& Ponsford, J. L. (2019). Cognitive behavioural therapy for post-stroke fatigue and sleep disturbance: a pilot randomised controlled trial with blind assessment. Neuropsychol Rehabil. 29(5), 723-738. https://doi. org/10.1080/09602011.2017.1326945. Epub 2017 May 19

98. Kim, C. R., Chun, M. H., \& Han, E. Y. (2010). Effects of hypnotics on sleep patterns and functional recovery of patients with subacute stroke. American Journal of Physical Medicine \& Rehabilitation, 89(4), 315-322.
99. Lee, S. H., \& Lim, S. M. (2016). Acupuncture for insomnia after stroke: A systematic review and meta-analysis. BMC Complementary and Alternative Medicine, 16, 228.

100. Merlino, G., Piani, A., Gigli, G. L., et al. (2010). Daytime sleepiness is associated with dementia and cognitive decline in older Italian adults: A population-based study. Sleep Medicine, 11(4), 372-377.

101. Rongve, A., Boeve, B. F., \& Arsland, D. (2010). Frequency and correlates of caregiver-reported sleep disturbances in a sample of persons with early dementia. Journal of the American Geriatrics Society, 58(3), 480-486.

102. Chen, P.-L., Lee, W.-J., Sun, W.-Z., Oyang, Y.-J., \& Fuh, J.-L. (2012). Risk of dementia in patients with insomnia and long-term use of hypnotics: A population-based retrospective cohort study. PLoS One, 7(11), e49113.

103. Johar, H., Kawan, R., Emeny, R. T., \& Ladwig, K. H. (2016). Impaired sleep predicts cognitive decline in old people: Findings from the prospective KORA age study. Sleep, 39(1), 217-226.

104. Shin, H.-Y., Han, H. J., Shin, D.-J., et al. (2014). Sleep problems associated with behavioral and psychological symptoms as well as cognitive functions in Alzheimer's disease. Journal of Clinical Neurology, 10(3), 203-209.

105. Kang, P., de Bruin, G. S., Wang, L. H., Ward, B. A., Ances, B. M., Lim, M. M., \& Bucelli, R. C. (2016). Sleep pathology in Creutzfeldt-Jakob disease. Journal of Clinical Sleep Medicine, 12(7), 1033-1039.

106. McCurry, S. M., Pike, K. C., Vitiello, M. V., et al. (2011). Increasing walking and bright light exposure to improve sleep in community-dwelling persons with Alzheimer's disease: Results of a randomized, controlled trial. Journal of the American Geriatrics Society, 59(8), 1393-1402.

107. Bjerre, L. M., Farrell, B., Hogl, M., et al. (2018). Deprescribing antipsychotics for behavioural and psychological symptoms of dementia and insomnia. Canadian Family Physician, 64, 17-27.

108. Declercq, T., Petrovic, M., Azermai, M., Vander, S. R., De Sutter, A. I., van Driel, M. L., et al. (2013). Withdrawal versus continuation of chronic antipsychotic drugs for behavioural and psychological symptoms in older people with dementia. Cochrane Database of Systematic Reviews, 3, CD007726.

109. McCleery, J., Cohen, D. A., \& Sharpley, A. L. (2016). Pharmacotherapies for sleep disturbances in dementia. Cochrane Database of Systematic Reviews, (11), CD009178

110. Lorenz, W., Ollenschlager, G., Geraedts, M., (Hrsg), et al. (2001). Das LeitlinienManual. Entwicklung und Implementierung von Leitlinien in der Medizin. Z Ärztl Fortbild Qualitätssich Gesundheitswes, 95, 1-84.

111. Sadeghi Bahmani, D., Esmaeili, L., Shaygannejad, V., Gerber, M., Kesselring, J., Lang, U. E., Holsboer-Trachsler, E., \& Brand, S. (2018). Stability of Mental Toughness, Sleep Disturbances, and Physical Activity in Patients With Multiple Sclerosis (MS)-A Longitudinal and Pilot Study. Front Psychiatry, 9, 182. https://doi.org/10.3389/fpsyt.2018.00182

\section{Publisher's Note}

Springer Nature remains neutral with regard to jurisdictional claims in published maps and institutional affiliations.

Ready to submit your research? Choose BMC and benefit from:

- fast, convenient online submission

- thorough peer review by experienced researchers in your field

- rapid publication on acceptance

- support for research data, including large and complex data types

- gold Open Access which fosters wider collaboration and increased citations

- maximum visibility for your research: over $100 \mathrm{M}$ website views per year

At $\mathrm{BMC}$, research is always in progress.

Learn more biomedcentral.com/submission 